\title{
Two-dimensional micromechanical bimorph arrays for detection of thermal radiation
}

\author{
S. R. Manalis, S. C. Minne, and C. F. Quate \\ E. L. Ginzton Laboratory, Stanford University, California 94305-4085 \\ G. G. Yaralioglu and A. Atalar \\ Bilkent University, Ankara, Turkey 06533
}

(Received 20 March 1997; accepted for publication 15 April 1997)

\begin{abstract}
We demonstrate that two-dimensional arrays of micromechanical bimorphs can be used as thermal sensors to image infrared (IR) radiation. A density of 100 pixels per $\mathrm{mm}^{2}$ is achieved by coiling a bimorph beam into the shape of a flat spiral. Temperature variations of a given spiral are converted to modulations of visible light by illuminating the spiral array with a visible source. The optical properties of the spiral resemble a Fresnel zone plate when light reflected off neighboring rings of the spiral is focused. When a spiral is heated through the absorption of IR radiation, thermally induced bending of the bimorph degrades the focusing efficiency by distorting the spiral. This reduces the optical intensity at the focal point. Arrays of spirals can be monitored with a commercial CCD camera. At $40 \mathrm{~Hz}$, the temperature resolution and noise equivalent power of a $75 \mu \mathrm{m}$ diam spiral are $50 \mu \mathrm{K} / \sqrt{ } \mathrm{Hz}$ and $20 \mathrm{nW} / \sqrt{ } \mathrm{Hz}$, respectively, and the thermal response time is $270 \mu \mathrm{s}$. (C) 1997 American Institute of Physics. [S0003-6951(97)03124-0]
\end{abstract}

Recent advances in uncooled detectors for infrared (IR) radiation have resulted in thermal imaging systems with excellent properties. The most recent systems achieve a sensitivity that approaches the thermal limit and operate at speeds of 60 frames/s. ${ }^{1-3}$ Images with high spatial resolution are obtained from arrays containing 400 pixels per $\mathrm{mm}^{2}$ by reading electronic signals generated at each detector. In order to process this information, multiple transistors are integrated into each pixel and a separate device is used to display the image. A direct view system where the thermal radiation is converted to a visible image is an alternative that does not require a complex readout system.

In this letter we describe a detection system consisting of micromechanical bimorphs that directly converts spatial variations of temperature to spatial variations of visible light. As an example, we use a two-dimensional array of bimorphs to create thermal images of a moving IR source. The work reported here has two features that distinguish it from other thermal imaging systems. First, it provides direct conversion from the IR to the visible and, second, it is a nonscanning system using a two-dimensional array of detectors spaced by $100 \mu \mathrm{m}$.

The use of a cantilever bimorph for thermal measurements was pioneered by Gimzewski et al. ${ }^{4}$ and by Barnes et $a .^{5}$ They demonstrated that microfabricated cantilevers could be coated with metal to form a bimorph and used for photothermal spectroscopy with a power resolution of $1 \mathrm{nW} /$ $\sqrt{ }$ Hz. This led to remote detection of thermal radiation, a possibility discussed by Oden et al., who pointed out that two-dimensional arrays of heat sensitive cantilevers can serve as thermal imaging devices. ${ }^{6,7}$ They demonstrated that bimorph cantilevers could be used to map the temperature distribution of a radiating object by scanning a single cantilever with an integrated piezoresistive sensor across the field.

In previous work, we found that a cantilever defined in the shape of interdigitated fingers could be used with an atomic force microscope (AFM) to measure displacements of $\sim 0.02 \AA{ }^{8}{ }^{8}$ Light is diffracted from the fingers into higher order modes. The intensity of a given mode is proportional to the displacement between alternate fingers. This feature makes it convenient to monitor the deflection of multiple cantilevers within a one-dimensional array. In the case of a two-dimensional array when a high spatial density of sensing elements is desired, the interdigitated fingers can be replaced by a single finger coiled into the shape of a spiral. The optical properties of the spiral resemble a Fresnel zone plate in that interference from light reflecting off neighboring rings of the spiral focuses the intensity at a point along the optical axis. When a spiral is heated through the absorption of IR radiation, thermally induced bending of the bimorph degrades the focusing efficiency which reduces the intensity at the focal point.

The diffraction of light from a spiral can be understood by first considering the behavior of a Fresnel zone plate. For simplicity, assume that the rings of the zone plate are created by depositing metal on a thin, optically transparent membrane. When illuminated with a coherent source, interference from light reflecting off each ring will concentrate roughly half of the reflected optical power at a focal point that is determined by the illumination wavelength and ring radii. ${ }^{9}$ When the temperature of the plate is altered, stress induced from differing thermal expansion coefficients of the metal rings and membrane will distort the plate. Distorting the plate by changing the temperature shifts the focal point along the axis normal to the spiral face. Distortion can then be monitored by measuring the intensity at a fixed point along the axis.

The spiral operates in a similar fashion as the zone plate. However, since spirals lack cylindrical symmetry, the exact description of their mechanical and optical properties is quite complicated. ${ }^{10}$ For example, the focal point of a spiral shifts along the axis as in the case of a zone plate, but also moves slightly off axis.

A scanning electron micrograph (SEM) of a bimorph spiral is shown in the inset of Fig. 1. The fabrication process begins by defining an array of spirals and a support grid with 


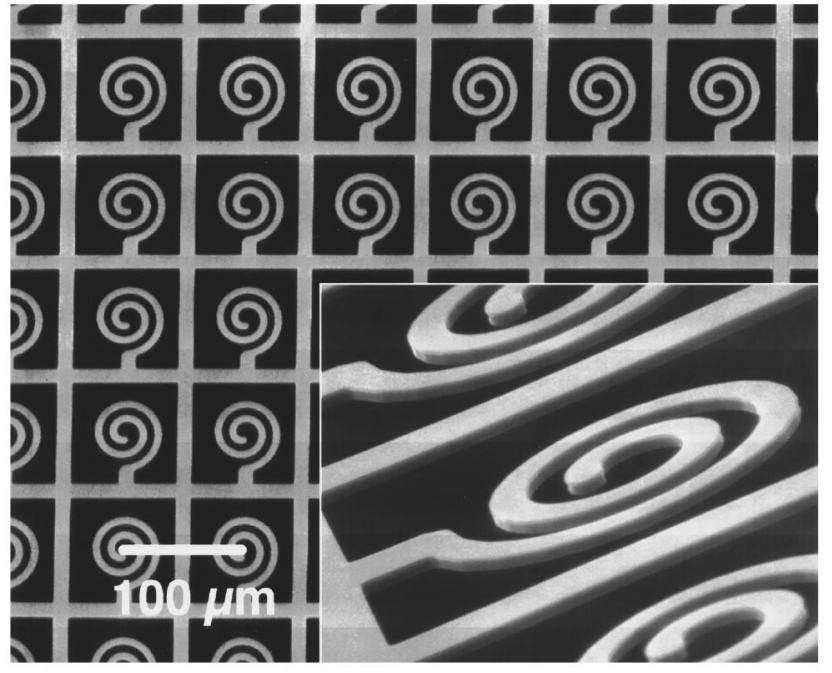

FIG. 1. SEM micrograph of a free-standing spiral array with a $96 \mu \mathrm{m}$ pitch. Inset: closeup of a single spiral.

a plasma etch on the top surface of a $\langle 100\rangle$ silicon-oninsulator wafer. The top surface is then passivated with polyimide and the bulk silicon is etched with ethylene diamine pyrocathecol (EDP) using the middle oxide as an etch stop. Both the spirals and support grid are released by etching the middle oxide in 6:1 buffered oxide etch (BOE) and removing the polyimide in an oxygen plasma. A bimorph is created by evaporating $200 \mathrm{~nm}$ of aluminum on the top silicon surface. This process was used to fabricate free-standing twodimensional spiral arrays with a pitch of 96,48 , and $24 \mu \mathrm{m}$ and a corresponding spiral arm width of 6,3 , and $1.5 \mu \mathrm{m}$. A SEM of an array with a $96 \mu \mathrm{m}$ pitch is shown in Fig. 1. The thickness of the top layer silicon is $1.5 \mu \mathrm{m}$ and the width of the support structure of the array is twice that of the spiral arm.

Diffraction patterns produced by a spiral can be imaged with the system shown in Fig. 2. The purpose of the optical imaging system is to translate the reflected diffraction pattern at the spiral focal plane to the CCD camera. A $630 \mathrm{~nm}$ visible laser diode with an output power of $<1 \mathrm{~mW}$ is used to illuminate the aluminum surface of the spiral with a collimated beam diameter of $3 \mathrm{~mm}$. The beam may also be focused on a single spiral by removing the lens used to focus the laser on the beam splitter.

Figure 3(a) shows the optical image from a $75 \mu \mathrm{m}$ diam spiral acquired at the spiral focal plane, which is located 4

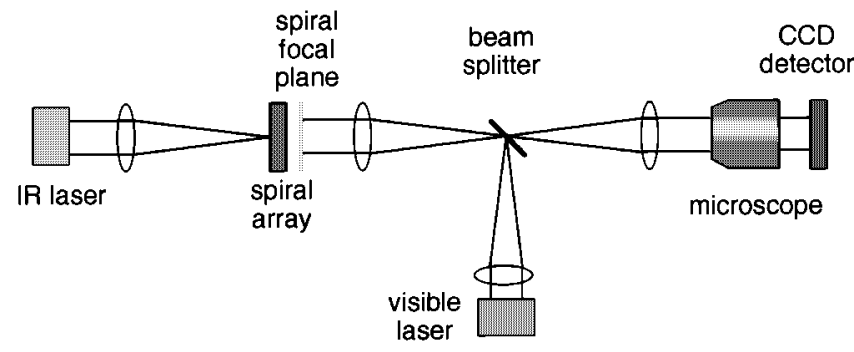

FIG. 2. Schematic of the optical imaging system used to project the spiral focal plane onto a CCD camera. The focal length of each lens is $65 \mathrm{~mm}$, and the array is illuminated with a $630 \mathrm{~nm}$ visible laser diode with a $3 \mathrm{~mm}$ beam diameter. Individual spirals within the arrays are heated from the back with a $780 \mathrm{~nm}$ IR laser diode.

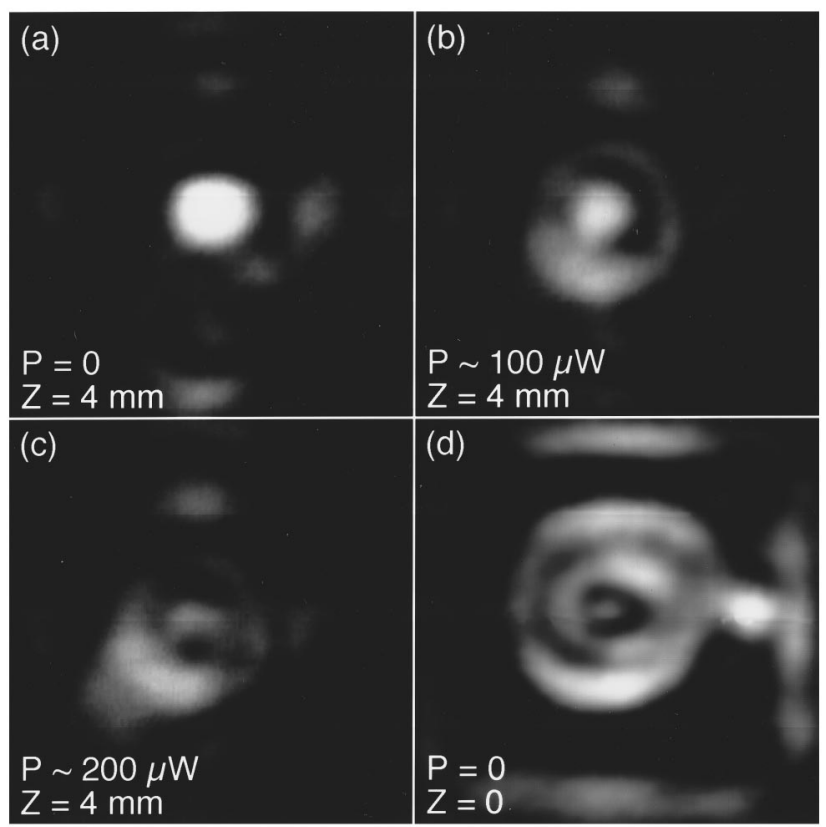

FIG. 3. Optical images of a $75 \mu \mathrm{m}$ diameter spiral at (a)-(c) the spiral focal plane which is located $4 \mathrm{~mm}$ above the spiral plane and (d) the spiral plane. In (b), $\sim 100 \mu \mathrm{W}$ of power from the IR laser is absorbed by the spiral. The resulting temperature change causes the spiral to distort and the focal point intensity is reduced. In (c), the focal point intensity reaches a minimum when the spiral absorbs $\sim 200 \mu \mathrm{W}$. These images were obtained without the visible laser focusing lens (shown in Fig. 2) in order to illuminate the spiral with $\mathrm{a} \sim 100 \mu \mathrm{m}$ beam diameter.

mm above the spiral plane. An individual spiral can be heated by focusing an IR laser on the back of the spiral. Figure 3(b) shows that the focal point intensity is reduced when the spiral absorbs roughly $100 \mu \mathrm{W}$ of power from a $780 \mathrm{~nm}$ diode laser. The absorbed power is estimated by assuming that most of the incident power is transmitted through the Si layer and that less than $10 \%$ is absorbed in the Al layer. The focal point intensity is minimized when the absorbed power is $\sim 200 \mu \mathrm{W}$ [see Fig. 3(c)] and does not change if the power is further increased. For reference, an image acquired at the spiral plane is shown in Fig. 3(d).

We can determine the temperature resolution and noise equivalent power (NEP) of the spiral by replacing the CCD camera with a split photodiode. One side of the split photodiode is used to measure the spiral focal point intensity while the other side is used to detect light surrounding the focal point. This allows noise from laser intensity fluctuations to be minimized by measuring the differential optical power. The thermal sensitivity is determined by changing the spiral and substrate temperature with a coil heater while recording the split photodiode output. The temperature of the spiral substrate is monitored with a thermistor. This yields a temperature resolution of $\sim 50 \mu \mathrm{K} / \sqrt{ } \mathrm{Hz}$ at a frequency of $40 \mathrm{~Hz}$. A NEP of $20 \mathrm{nW} / \sqrt{ } \mathrm{Hz}$ and a thermal response time of $270 \mu \mathrm{s}$ is measured by heating the spiral with the IR laser diode. If we assume that our device absorbs $10 \%$ of the incident radiation, then the minimum detectable power is $2 \mathrm{nW} / \sqrt{\mathrm{Hz}}$. For comparison, the temperature resolution, NEP, and response time of a commercial bolometric IR detector with a $46 \mu \mathrm{m}$ pixel size is roughly $20 \mu \mathrm{K} / \sqrt{ } \mathrm{Hz}, 0.004 \mathrm{nW} / \sqrt{ } \mathrm{Hz}$, and $12 \mathrm{~ms}$, respectively. ${ }^{2}$ Although the temperature resolution of 
(a)

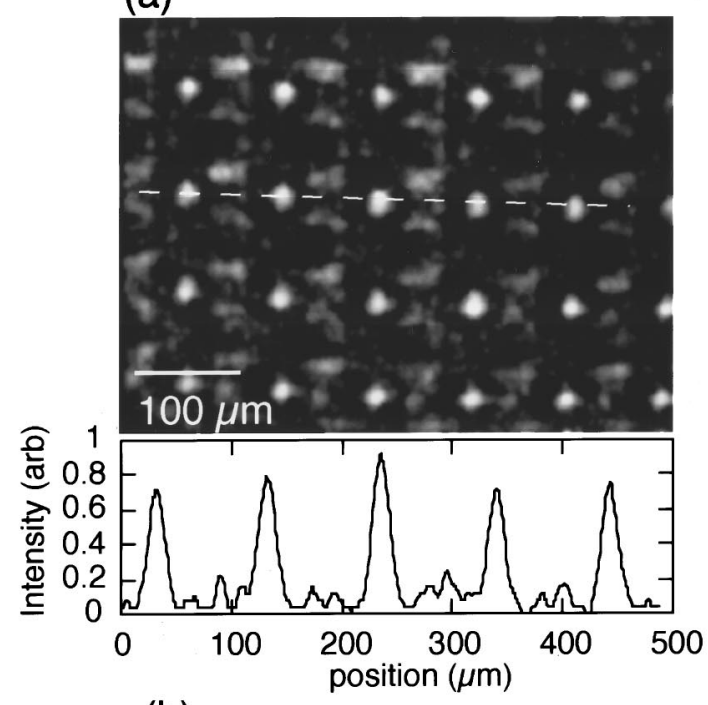

(b)

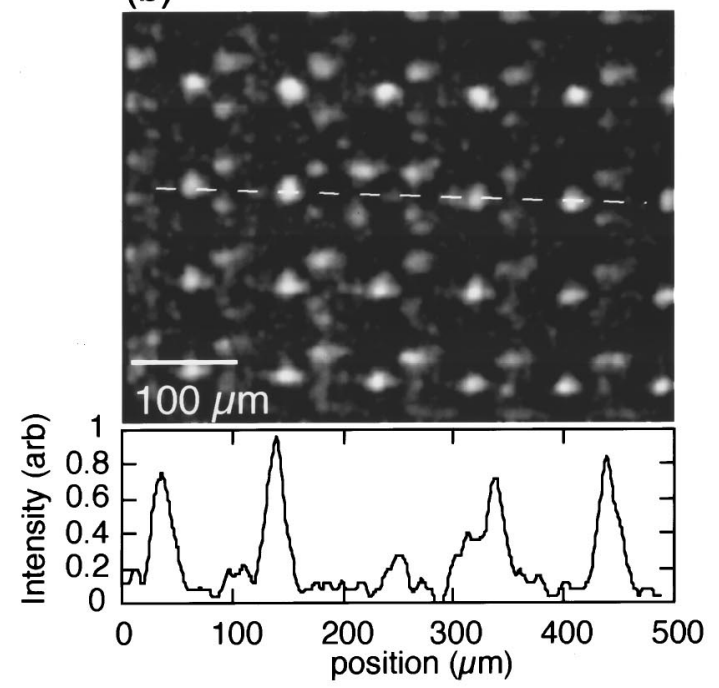

FIG. 4. Optical image at the spiral focal plane of a $6 \times 4$ array obtained when (a) the IR heating source is off and (b) $\sim 200 \mu \mathrm{W}$ of power is absorbed by one of the center spirals. A cross section of the intensity profile along the dotted line indicates that the focal point intensity of the heated spiral is significantly reduced.

our device is comparable to this detector, our NEP is significantly higher. Since the thermal response time of our device is much faster than in necessary for an operation rate of 60 frames/s, the NEP may be improved by decreasing the thermal conductance between the spiral and support grid. This could be accomplished by reducing the thickness of the bimorph and minimizing the width of the spiral support stem. The NEP can also be improved by depositing an IR absorber coating such as platinum on the back surface of the spiral.

The focal plane from multiple spirals can also be imaged with the system shown in Fig. 2. An image acquired at the focal plane of an array of spirals spaced by $96 \mu \mathrm{m}$ is shown in Fig. 4(a). Shown below is a cross section of the optical intensity distribution along the dotted line. Surrounding each focal spot is unwanted optical interference from light reflecting off the spiral support grid. In Fig. 4(b), $\sim 200 \mu \mathrm{W}$ of IR power is absorbed by one of the center spirals. A comparison between the cross section of the optical intensity distribution along the dotted line of Figs. 4(a) and 4(b) indicates that the focal spot of the heated spiral is significantly reduced while peaks from neighboring spirals are slightly affected. This coupling is likely caused by optical interference originating from movement of the support grid. ${ }^{11}$ To demonstrate the imaging capability of the spiral array, we translated the IR heating source over various spirals within the array shown in Fig. 4 while capturing a sequence of frames. The position of the IR source could then be tracked by monitoring the focal point intensity of a given spiral.

We have described a thermal imaging system where spatially varying temperature distributions are directly converted to spatially varying intensity distributions of visible light. This system eliminates the need to integrate readout circuity with the thermal sensors since it allows an image to be viewed directly. Since the thermal sensors are detected remotely, they should be able to function in a wide range of chemical environments and can be easily replaced if contaminated while still preserving the optical imaging system. For example, we believe that a direct view system can also be implemented by depositing reflecting Fresnel zone plates on thermally isolated membranes made of silicon nitride.

The authors would like to thank Tom Kenny, Ken Goodson, Nick Ulman, Yongho Sungtaek Ju, Dave Dickensheets, and Texas Instruments for providing valuable discussions. The primary support for this work came from the Joint Services Electronics Program N00014-91-J-1050.

${ }^{1}$ P. K. Kruse, Proc. SPIE IV, 34 (1996).

${ }^{2}$ C. Marshall, N. Butler, R. Blackwell, R. Murphy, and T. Breen, Proc. SPIE IV, 23 (1996).

${ }^{3}$ W. Radford, D. Murphy, M. Ray, S. Propst, A. Kennedy, J. Kojiro, J. Woolaway, and K. Soch, Proc. SPIE IV, 82 (1996).

${ }^{4}$ J. K. Gimzewski, Ch. Gerber, E. Meyer, and R. R. Schlittler, Chem. Phys. Lett. 217, 589 (1994).

${ }^{5}$ J. R. Barnes, R. J. Stephenson, M. E. Welland, Ch. Gerber, and J. K. Gimzewski, Nature (London) 372, 79 (1994).

${ }^{6}$ P. G. Datskos, P. I. Oden, T. Thundat, E. A. Wachter, R. J. Warmack, and S. R. Hunter, Appl. Phys. Lett. 69, 2986 (1996).

${ }^{7}$ P. I. Oden, P. G. Datskos, T. Thundat, and R. J. Warmack, Appl. Phys. Lett. 69, 3277 (1996).

${ }^{8}$ S. R. Manalis, S. C. Minne, A. Atalar, and C. F. Quate, Appl. Phys. Lett. 69, 3944 (1996).

${ }^{9}$ E. Hecht, Optics, 2nd ed. (Addison-Wesley, Reading, 1987), p. 445.

${ }^{10}$ We have analyzed the mechanical bending of a bimorph spiral using ANSYS simulation software.

${ }^{11}$ The support grid spans an area of nearly $1 \mathrm{~mm}^{2}$ and is less than $2 \mu \mathrm{m}$ thick. Increasing the thickness will improve both the temperature and mechanical stability of the support grid. The optical reflectivity of the grid can be reduced by depositing metal only on the spirals. 